\title{
LAPLACE TRANSFORM SOLUTION OF THE PROBLEM OF TIME-FRACTIONAL HEAT CONDUCTION IN A TWO-LAYERED SLAB
}

\author{
Stanistaw Kukla, Urszula Siedlecka \\ Institute of Mathematics, Czestochowa University of Technology \\ Częstochowa, Poland \\ stanislaw.kukla@im.pcz.pl,urszula.siedlecka@im.pcz.pl
}

\begin{abstract}
In this paper the Laplace transformation for solving the problem of fractional heat conduction in a two-layered slab has been applied. The different orders of Caputo derivative in the time-fractional equation governed the heat transfer in the layers are assumed. The inverse Laplace transform by using a numerical method is determined. The numerical results obtained by using of the eigenfunctions method and by numerically inverting the Laplace transform are compared.
\end{abstract}

Keywords: fractional heat conduction, two-layered slab, Laplace transformation

\section{Introduction}

Classical models of the heat conduction are derived with assumption of the Fourier law of heat transfer. The heat conduction problems in multi-layered bodies based on the Fourier law is the subject of paper [1] by Haji-Sheikh and Beck. Exact solutions of these problems for multi-layered slabs, cylinders and spheres are presented by Özişik in book [2].

A generalization of the Fourier law leads to fractional heat conduction. The fractional heat equation includes a fractional derivative with respect to space and/or time variable. The problems of fractional heat conduction are the subject of numerous works, for instance references [3-7]. In book [3] by Povstenko the equations obtained by generalizations of the time-nonlocal Fourier's, Fick's and Darcy's laws are discussed. The equations in Cartesian, polar, cylindrical and spherical coordinates are considered. The problems of heat conduction in a semiinfinite or an infinite composite medium consisting of two regions characterizing by different orders of the time-fractional Caputo derivative in the heat equation were studied by Povstenko in papers [4-7]. The presented solutions are derived with assumption that the two considered solids are in perfect thermal contact. The heat conduction in a multi-layer slab governed by a time-fractional equation is discussed by Siedlecka and Kukla in reference [8]. The classical convective bound- 
ary conditions and the classical conditions describing the perfect contact of the solids were assumed. To solving the problem the eigenfunctions method was applied. Application of the Laplace transformation to solving the fractional differential equation is shown by Podlubny in the book [9]. The inverse of the Laplace transform can be determined numerically. The methods for numerical inversion of the Laplace transforms are presented in papers $[10,11]$.

In this paper, a solution of the time-fractional heat conduction in a two-layered slab is presented. The physical Robin condition on the sphere surface and the perfect contact of the layers are assumed. The Laplace transformation is applied and the temperature in the slab is obtained by using a method of numerical inversion of the Laplace transforms.

\section{Formulation of the problem}

Consider a slab consisting of two layers with thermal conductivity $\lambda_{i}$ and thermal diffusivity $a_{i}$. The time-fractional differential equation of the heat conduction in the $i$-th layer is

$$
\frac{\partial^{2} T_{i}}{\partial x^{2}}+\frac{1}{\lambda_{i}} q_{i}(x, t)=\frac{1}{a_{i}} \frac{\partial^{\alpha_{i}} T_{i}}{\partial t^{\alpha_{i}}}, \quad x \in\left[x_{i-1}, x_{i}\right], \quad 0<\alpha_{i} \leq 1, \quad i=1,2
$$

where $T_{i}(x, t)$ is a temperature in the $i$-th layer and $q_{i}(x, t)$ is a volumetric energy generation, $x_{0}=0, x_{2}$ and $x_{1}$ are coordinates specifying surfaces of the slab boundaries and an interface between the layers, respectively, $\alpha_{i}$ denotes an order of the Caputo fractional derivative with respect to time $t$. The Caputo derivative of order $\alpha$ is defined by [9]

$$
{ }_{0}^{C} D_{t}^{\alpha} f(t)=\frac{d^{\alpha} f(t)}{d t^{\alpha}}=\frac{1}{\Gamma(m-\alpha)} \int_{0}^{t}(t-\tau)^{m-\alpha-1} \frac{d^{m} f(\tau)}{d \tau^{m}} d \tau, \quad m-1<\alpha<m
$$

We assume the Robin boundary conditions [3] at $x=0$ and $x=x_{2}$

$$
\begin{gathered}
\lambda_{1}^{*} D_{R L}^{1-\alpha_{1}} \frac{\partial T_{1}}{\partial x}\left(x_{0}, t\right)=-a_{L}\left(T_{L}(t)-T_{1}\left(x_{0}, t\right)\right) \\
-\lambda_{2}^{*} D_{R L}^{1-\alpha_{2}} \frac{\partial T_{2}}{\partial x}\left(x_{2}, t\right)=-a_{R}\left(T_{R}(t)-T_{2}\left(x_{2}, t\right)\right)
\end{gathered}
$$

where $a_{L}, a_{R}$ are heat transfer coefficients, $T_{L}(t), T_{R}(t)$ are surrounding temperatures and $D_{R L}^{\alpha}$ denotes the Riemann-Liouville fractional derivative of order $\alpha$. Moreover, the conditions of the perfect contact of the layers are satisfied [3] 


$$
\begin{gathered}
T_{1}\left(x_{1}, t\right)=T_{2}\left(x_{1}, t\right) \\
\lambda_{1} D_{R L}^{1-\alpha_{1}} \frac{\partial T_{1}}{\partial x}\left(x_{1}, t\right)=\lambda_{2} D_{R L}^{1-\alpha_{2}} \frac{\partial T_{2}}{\partial x}\left(x_{1}, t\right)
\end{gathered}
$$

and the initial condition is

$$
T_{i}(x, 0)=f_{i}(x), \quad x \in\left[x_{i-1}, x_{i}\right], \quad i=1,2
$$

The Riemann-Liouville fractional derivative occurring in equations (3), (4) and (6) is defined by [9]

$$
D_{R L}^{\alpha} f(t)=\frac{1}{\Gamma(m-\alpha)} \frac{d^{m}}{d t^{m}} \int_{0}^{t}(t-\tau)^{m-\alpha-1} f(\tau) d \tau, \quad m-1<\alpha<m
$$

The conditions (3), (4) of the convective heat transfer between the slab and the surroundings are called the physical Robin conditions [3]. Substituting $\alpha_{1}=\alpha_{2}=1$ in equations (3), (4) the classical conditions of the third kind are obtained. These conditions in the theory of fractional heat transfer are also called mathematical Robin conditions [3]. In this case the condition (6) of equality of the heat fluxes at the interface assumes also a classical form. A solution of the heat conduction problem in the slab under the physical Robin boundary conditions by using the Laplace transformation is presented in Section 3 and a solution of the problem under the mathematical Robin boundary conditions applying the eigenfunctions method is shown in Section 4.

\section{Application of the Laplace transformation}

In order to solve the problem (1)-(7) we use the Laplace transformation with respect to time $t$ which is defined by

$$
\bar{F}(s)=\int_{0}^{\infty} F(t) e^{-s t} d t
$$

where $s$ is a complex parameter. The following property of the Laplace transformation of a fractional derivative will be used [4]

$$
L\left[\frac{d^{\alpha} F(t)}{d t^{\alpha}}\right]=s^{\alpha} \bar{F}(s)-s^{\alpha-1} F(0)
$$

where $0<\alpha \leq 1$. Applying the Laplace transformation to equation (1) and using the property (10), we obtain an ordinary differential equation in the form 
$\frac{d^{2} \bar{T}_{i}}{d x^{2}}-\frac{s^{\alpha_{i}}}{a_{i}} \bar{T}_{i}=-\frac{s^{\alpha_{i}-1}}{a_{i}} f_{i}(x)-\frac{1}{\lambda_{i}} \bar{q}_{i}(x, s), \quad x \in\left[x_{i-1}, x_{i}\right], \quad 0<\alpha_{i} \leq 1, \quad i=1,2$

The boundary conditions (3), (4) in the transform domain are

$$
\begin{gathered}
\lambda_{1}^{*} s^{1-\alpha_{1}} \frac{d \bar{T}_{1}}{d x}(0, s)=-a_{L}\left(\bar{T}_{L}(s)-\bar{T}_{1}(0, s)\right) \\
\lambda_{1}^{*} s^{1-\alpha_{2}} \frac{d \bar{T}_{2}}{d x}\left(x_{2}, s\right)=a_{R}\left(\bar{T}_{R}(s)-\bar{T}_{2}\left(x_{2}, s\right)\right)
\end{gathered}
$$

and the conditions (5), (6) assume the form

$$
\begin{aligned}
\bar{T}_{1}\left(x_{1}, s\right) & =\bar{T}_{2}\left(x_{1}, s\right) \\
\lambda_{1} s^{1-\alpha_{1}} \frac{d \bar{T}_{1}}{d x}\left(x_{1}, s\right) & =\lambda_{2} s^{1-\alpha_{2}} \frac{d \bar{T}_{2}}{d x}\left(x_{1}, s\right)
\end{aligned}
$$

The general solution of the equation (11) can be written in the form

$$
\bar{T}_{i}(x, s)=C_{i 1} e^{-P_{i} x}+C_{i 2} e^{P_{i} x}+\frac{1}{P_{i}} \int_{x_{i-1}}^{x} R_{i}(\xi, s) \cdot \sinh \left(P_{i} \cdot(x-\xi)\right) d \xi
$$

where $P_{i}=\sqrt{\frac{s^{\alpha_{i}}}{a_{i}}}$ and $R_{i}(\xi, s)=-\frac{s^{\alpha_{i}-1}}{a_{i}} f_{i}(\xi)-\frac{1}{\lambda_{i}} \bar{q}_{i}(\xi, s)$. The constants $C_{i 1}, C_{i 2}$ are determined by using the conditions (12)-(15). Assuming: $f_{i}(x)=0$ and $q_{i}(x, t)=0$ for $i=1,2$, and solving the system of four equations, we obtain the constants $C_{11}, C_{12}, C_{21}, C_{22}$. After transformation, we get

$$
\begin{aligned}
C_{11} & =\left(\frac{1}{2} \bar{T}_{L}(s)\left(e^{P_{1} x_{1}-2 P_{2} x_{2}}\left(1-w_{21}\right)\left(1-w_{2 R}\right)-e^{\left(P_{1}-2 P_{2}\right) x_{1}}\left(1+w_{21}\right)\left(1+w_{2 R}\right)\right)+\right. \\
& \left.-\bar{T}_{R}(s) e^{-P_{2}\left(x_{1}+x_{2}\right)} w_{21}\left(1-w_{1 L}\right)\right) / M \\
C_{12} & =\left(-\frac{1}{2} \bar{T}_{L}(s)\left(e^{-\left(P_{1}+2 P_{2}\right) x_{1}}\left(1-w_{21}\right)\left(1+w_{2 R}\right)-e^{-P_{1} x_{1}-2 P_{2} x_{2}}\left(1+w_{21}\right)\left(1-w_{2 R}\right)\right)-\right. \\
& \left.-\bar{T}_{R}(s) e^{-P_{2}\left(x_{1}+x_{2}\right)} w_{21}\left(1+w_{1 L}\right)\right) / M
\end{aligned}
$$




$$
\begin{aligned}
C_{21}= & \left(-\bar{T}_{L}(s) e^{-P_{2} x_{1}}\left(1+w_{2 R}\right)+\right. \\
& \left.\bar{T}_{R}(s) e^{-P_{2} x_{2}}\left(\left(w_{1 L}-w_{21}\right) \sinh P_{1} x_{1}+\left(1-w_{21} w_{1 L}\right) \cosh P_{1} x_{1}\right)\right) / M \\
C_{22}= & \left(\bar{T}_{L}(s) e^{-P_{2}\left(x_{1}+2 x_{2}\right)}\left(1-w_{2 R}\right)-\right. \\
& \left.\bar{T}_{R}(s) e^{-P_{2}\left(2 x_{1}+x_{2}\right)}\left(\left(1+w_{1 L} w_{21}\right) \cosh P_{1} x_{1}+\left(w_{1 L}+w_{21}\right) \sinh P_{1} x_{1}\right)\right) / M
\end{aligned}
$$

where $w_{1 L}=\frac{P_{1} \lambda_{1}^{*}}{a_{L}} s^{1-\alpha_{1}}, w_{2 L}=\frac{P_{2} \lambda_{2} \lambda_{1}^{*}}{a_{L} \lambda_{1}} s^{1-\alpha_{2}}, w_{2 R}=\frac{P_{2} \lambda_{2}^{*}}{a_{R}} s^{1-\alpha_{2}}, w_{21}=\frac{P_{2} \lambda_{2}}{P_{1} \lambda_{1}} s^{\alpha_{1}-\alpha_{2}}$ and

$$
\begin{aligned}
M= & \left(-e^{-2 P_{2} x_{1}}\left(1+w_{2 R}\right)\left(1+w_{2 L}\right)+e^{-2 P_{2} x_{2}}\left(1-w_{2 R}\right)\left(1-w_{2 L}\right)\right) \cosh P_{1} x_{1}+ \\
& \left(-e^{-2 P_{2} x_{1}}\left(w_{1 L}+w_{21}\right)\left(1+w_{2 R}\right)+e^{-2 P_{2} x_{2}}\left(w_{1 L}-w_{21}\right)\left(1-w_{2 R}\right)\right) \sinh P_{1} x_{1}
\end{aligned}
$$

The Laplace transform (16) cannot be inverted analytically and that is why numerical methods for inversion must be applied. In the next section, the solution for a case of the heat conduction problem by applying the eigenfunctions method is presented and in the following section the numerical results obtained by using both methods are compared.

\section{The method of eigenfunctions}

A solution to the problem of the heat conduction in a multi-layered slab with a fractional time-derivative of the same order in each layer under the mathematical Robin boundary conditions is presented by Siedlecka and Kukla in the paper [8]. The temperature distribution in the slab by using the method of eigenfunctions has been obtained. In the case of the heat conduction in a two-layer slab, the formula for temperature in the layers can be rewritten in the following form:

$$
T_{i}(x, t)=\theta_{i}(x, t)+\Phi_{i}^{1}(x) T_{L}(t)+\Phi_{i}^{2}(x) T_{R}(t), \quad i=1,2
$$

where

$$
\theta_{i}(x, t)=\sum_{k=1}^{\infty} \Gamma_{k}(t) \Psi_{i, k}(x), \quad x_{i-1} \leq x \leq x_{i}, \quad i=1,2
$$

and 


$$
\begin{array}{ll}
\Phi_{1}^{1}(x)=-\frac{1}{H} \frac{\lambda_{2}}{\lambda_{1}}\left(\frac{\lambda_{1}^{*}}{a_{L}}+x\right)+1 & \Phi_{2}^{1}(x)=\frac{1}{H}\left(\frac{\lambda_{2}^{*}}{a_{R}}+x_{2}-x\right) \\
\Phi_{1}^{2}(x)=\frac{1}{H} \frac{\lambda_{2}}{\lambda_{1}}\left(\frac{\lambda_{1}^{*}}{a_{L}}+x\right) & \Phi_{2}^{2}(x)=-\frac{1}{H}\left(\frac{\lambda_{2}^{*}}{a_{R}}+x_{2}-x\right)+1
\end{array}
$$

where $H=\left(\frac{\lambda_{1}^{*}}{a_{L}}+x_{1}\right) \frac{\lambda_{2}}{\lambda_{1}}+\frac{\lambda_{2}^{*}}{a_{R}}+x_{2}-x_{1}$. The functions $\Gamma_{k}$ in equation (18) are given by

$$
\begin{aligned}
& \Gamma_{k}(t)=\frac{E_{\alpha}\left(-\beta_{k}^{2} t^{\alpha}\right)}{N_{k}} \sum_{i=1}^{2} \frac{\lambda_{i}}{a_{i}} \int_{x_{i-1}}^{x_{i}} \tilde{f}_{i}(x) \Psi_{i, k}(x) d x+ \\
& +\frac{1}{N_{k}} \int_{0}^{t}(t-\tau)^{\alpha-1} E_{\alpha, \alpha}\left(-\beta_{k}^{2}(t-\tau)^{\alpha}\right) \sum_{i=1}^{2} \int_{x_{i-1}}^{x_{i}} \tilde{q}_{i}(x, \tau) \Psi_{i, k}(x) d x d \tau
\end{aligned}
$$

where $E_{\alpha, \beta}$ is the Mittag-Leffler function [12], $E_{\alpha}=E_{\alpha, 1}$ and

$$
\begin{gathered}
\tilde{f}_{i}(x)=f_{i}(x)-\Phi_{i}^{1}(x) T_{L}(0)-\Phi_{i}^{2}(x) T_{R}(0) \\
\tilde{q}_{i}(x, t)=q_{i}(x, t)-\frac{\lambda_{i}}{a_{i}} \frac{d^{\alpha} T_{L}}{d t^{\alpha}} \Phi_{i}^{1}(x)-\frac{\lambda_{i}}{a_{i}} \frac{d^{\alpha} T_{R}}{d t^{\alpha}} \Phi_{i}^{2}(x)
\end{gathered}
$$

The functions $\Psi_{i, k}$ occurring in equation (18) are given by

$$
\begin{aligned}
& \Psi_{1, k}(x)=\frac{\beta_{1, k} \lambda_{1}^{*}}{a_{L}} \cos \beta_{i, k} x+\sin \beta_{1, k} x \\
& \Psi_{2, k}(x)=A_{k} \cos \beta_{2, k}\left(x-x_{1}\right)-\frac{\beta_{1, k} \lambda_{1}}{\beta_{2, k} \lambda_{2}} B_{k} \sin \beta_{2, k}\left(x-x_{1}\right)
\end{aligned}
$$

where $A_{k}=\frac{\beta_{1, k} \lambda_{1}^{*}}{a_{L}} \cos \beta_{1, k} x_{1}+\sin \beta_{1, k} x_{1}, \quad B_{k}=\frac{\beta_{1, k} \lambda_{1}}{\beta_{2, k} \lambda_{2}}\left(\frac{\beta_{1, k} \lambda_{1}^{*}}{a_{L}} \sin \beta_{1, k} x_{1}-\cos \beta_{1, k} x_{1}\right)$ and $\beta_{i, k}=\gamma_{k} / \sqrt{a_{i}}$, wherein $\gamma_{k}$ is $k$-th root of the eigenvalue equation

$$
\left(A_{k}-\frac{\beta_{2, k} \lambda_{2}^{*}}{a_{R}} B_{k}\right) \cos \beta_{2, k}\left(x_{2}-x_{1}\right)-\left(\frac{\beta_{2, k} \lambda_{2}^{*}}{a_{R}} A_{k}+B_{k}\right) \sin \beta_{2, k}\left(x_{2}-x_{1}\right)=0
$$


The coefficients $N_{k}$ occurring in equation (20) are given by

$$
\begin{aligned}
N_{k}= & \frac{\lambda_{1}}{a_{1} \beta_{1, k}}\left(\beta_{1, k} x_{1}\left(1+u_{k}^{2}\right)+u_{k}\left(1-\cos \left(2 \beta_{1, k} x_{1}\right)\right)-\frac{1}{2}\left(1-u_{k}^{2}\right) \sin \left(2 \beta_{1, k} x_{1}\right)\right)+ \\
& +\frac{B_{k}^{2} \lambda_{2}}{a_{2} \beta_{2, k}}\left(\left(1+V_{k}^{2}\right) d_{k}+V_{k}\left(1-\cos \left(2 d_{k}\right)\right)\right)-\left(1-V_{k}^{2}\right) \sin \left(2 d_{k}\right)
\end{aligned}
$$

where $d_{k}=\left(x_{2}-x_{1}\right) \beta_{2, k}, u_{k}=\frac{\beta_{1, k} \lambda_{1}^{*}}{a_{L}}$ and $V_{k}=\frac{A_{k}}{B_{k}}$.

The temperature distribution in the two-layered slab is completely specified by equations (17)-(21) and (23) where the eigenvalues $\gamma_{k}$ are roots of equation (22).

\section{Numerical examples}

The Laplace transform of the temperature distribution in a two-layered slab for different models of the fractional heat conduction is given by equation (16). The inverse Laplace transform will be obtained numerically. For numerical inversion of the Laplace transforms a method will be applied which used the following formula [10]

$$
f(t)=\lim _{n \rightarrow \infty} f_{n}(t)
$$

where $f_{n}(t)=\frac{n \log 2}{t}\left(\begin{array}{c}2 n \\ n\end{array}\right) \sum_{j=0}^{n}(-1)^{j}\left(\begin{array}{c}n \\ j\end{array}\right) \bar{f}\left((n+j) \frac{\log 2}{t}\right)$.

The results obtained by numerical inversion of the Laplace transform are compared with the results computed by using the formula which is obtained by the method of eigenfunctions for the case of heat conduction in the two-layered slab under the mathematical Robin boundary conductions. The numerical calculations were performed for the slab, whose outer boundaries are at: $x_{0}=0, x_{2}=0.4 \mathrm{~m}$ and the interface is at $x_{1}=0.2 \mathrm{~m}$. The heat transfer coefficients are assumed as: $a_{L}=1200.0, a_{R}=600.0 \mathrm{~W} /\left(\mathrm{m}^{2} \cdot{ }^{\circ} \mathrm{C}\right)$, the thermal diffusivities: $a_{1}=3.35 \times 10^{-6}$, $a_{2}=5.42 \times 10^{-6} \mathrm{~m}^{2} / \mathrm{s}^{\alpha}$, and the thermal conductivities: $\lambda_{1}=16.0, \lambda_{2}=24.0$ $\mathrm{W} /\left(\mathrm{m} \cdot{ }^{\circ} \mathrm{C}\right)$. The initial temperature $T_{0}$ and the ambient temperature $T_{R}$ were constants: $T_{0}=0, T_{R}=0$. The ambient temperature $T_{L}$ was a function of time: $T_{L}(t)=A+B \sin v t$, where $A=100^{\circ} \mathrm{C}, B=50^{\circ} \mathrm{C}$ and $v=2 \pi / 5000 \mathrm{~s}^{-1}$. Numerical calculations were carried out using the Mathematica package. 
Table 1

Comparison of temperatures in the slab at $x=x_{1}$ for various values of time $\tilde{t}$ and order $\alpha$ obtained by numerically inverting Laplace transform and by eigenfunctions method

\begin{tabular}{|c|c|c|c|}
\hline \multirow{2}{*}{$\tilde{t}$} & $\alpha$ & \multicolumn{2}{|c|}{ Temperature $\left[{ }^{\circ} \mathrm{C}\right]$} \\
\cline { 2 - 4 } & & Method of Laplace transform & Method of eigenfunctions \\
\hline \multirow{3}{*}{100} & 0.8 & 10.3947 & 10.3282 \\
\cline { 2 - 4 } & 0.9 & 27.4455 & 26.9785 \\
\cline { 2 - 4 } & 1.0 & 41.3265 & 40.9439 \\
\hline \multirow{3}{*}{110} & 0.8 & 11.4831 & 11.5006 \\
\cline { 2 - 4 } & 0.9 & 28.9300 & 29.4724 \\
\cline { 2 - 4 } & 1.0 & 41.7893 & 44.0471 \\
\hline \multirow{3}{*}{120} & 0.8 & 12.5203 & 12.5679 \\
\cline { 2 - 4 } & 0.9 & 30.2325 & 30.1093 \\
\cline { 2 - 4 } & 1.0 & 42.1109 & 40.0181 \\
\hline
\end{tabular}

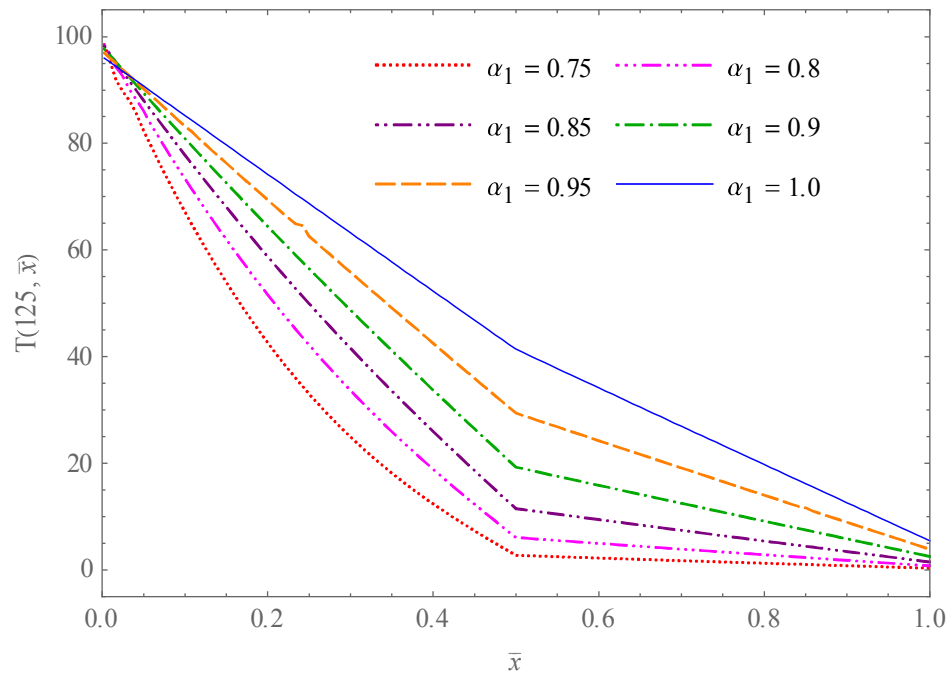

Fig. 1. Temperature in the slab as a function of $\tilde{x}=x / x_{2}$ at the non-dimensional time $\tilde{t}=125$ for different orders $\alpha_{1}=0,75 ; 0,85 ; 0,9 ; 0,95 ; 1,0$ and $\alpha_{2}=1.0$

The temperatures of the slab at $x=x_{1}$ computed by using the method of the Laplace transform and the method of eigenfunctions for various values of the order $\alpha$ (constant and the same in both layers) and for various non-dimensional time $\tilde{t}=t \cdot x_{2} / \sqrt{a_{2}}$ are tabulated in Table 1. Comparison of the temperatures shows a good agreement of the results obtained by using the two methods. 
The temperature distribution in the two-layered slab under the physical Robin boundary conditions as a function of $\tilde{x}=x / x_{2}$ at time $\tilde{t}=125$ is presented in Figure 1. The heat conduction in the layers is characterized by various orders of time-derivative in the heat equation: $\alpha_{1}=0,75 ; 0,85 ; 0,9 ; 0,95 ; 1,0$ and $\alpha_{2}=1.0$. The remaining data are the same as those presented above. The curves in Figure 1 show that the ratio of the fractional orders in the layers is significant to the heat conduction process in the slab.

\section{Conclusions}

The fractional heat conduction in a two-layered slab under the physical Robin boundary conditions was considered. A solution of the problem by the Laplace transformation has been obtained. The inverse of the Laplace transform was numerically determined. Good agreement shows a comparison of the numerical results obtained by numerically inverting the Laplace transform and by the method of eigenfunctions applying to the heat conduction problem in a slab under the mathematical Robin boundary conditions. Because the numerical inversion of the Laplace transform is an ill conditioned problem, an application of a testing method is required. Although the presented results referred to the fractional heat conduction in the two-layered slab, the method can be applied to the problems of the fractional heat conduction in multi-layered slabs.

\section{References}

[1] Haji-Sheikh A., Beck J. V., Temperature solution in multi-dimensional multi-layer bodies, International Journal of Heat and Mass Transfer 2002, 45, 1865-1877.

[2] Özişik M. N., Heat Conduction, Wiley, New York 1993.

[3] Povstenko Y., Linear Fractional Diffusion-wave Equation for Scientists and Engineers, Birkhauser, New York 2015.

[4] Povstenko Y., Fractional heat conduction in a semi-infinite composite body, Communications in Applied and Industrial Mathematics 2014, 6, 1, e-482.

[5] Povstenko Y., Fundamental solutions to time-fractional heat conduction equations in two joint half-lines, Central European Journal of Physics 2013, 11, 1284-1294.

[6] Povstenko Y., Fractional heat conduction in an infinite medium with a spherical inclusion, Entropy 2013, 15, 4122-4133.

[7] Povstenko Y., Fractional heat conduction in infinite one-dimensional composite medium. Journal of Thermal Stresses 2013, 36, 351-363.

[8] Siedlecka U., Kukla S., A solution to the problem of time-fractional heat conduction in a multilayer slab, Journal of Applied Mathematics and Computational Mechanics 2015, 14(3), 95-102.

[9] Podlubny I., Fractional Differential Equations, Academic Press, San Diego 1999.

[10] Valko P.P., Abate J., Numerical inversion of 2-D Laplace transforms applied to fractional diffusion equations, Applied Numerical Mathematics 2005, 53, 73-88.

[11] Wang Q., Zhan H., On different numerical inverse Laplace methods for solute transport problems, Advances in Water Resources 2015, 75, 80-92.

[12] Povstenko Y., Klekot J., The Dirichlet problem for time-fractional advection-diffusion equation in a half-space, Journal of Applied Mathematics and Computational Mechanics 2015, 14(2), 73-83. 